\title{
APPLICATIONS OF TERAHERTZ ATR SPECTROSCOPY TO PHARMACOLOGY
}

\author{
EDWARD FRANCISZEK PLIŃSKI ${ }^{1 *}$, NORBERT PAŁKA² ${ }^{2}$, and STANISŁAWA PLIŃSKA ${ }^{3}$
}

${ }^{1}$ Faculty of Electronics, Wroclaw University of Science and Technology, 50-370 Wroclaw, Poland

${ }^{2}$ Institute of Optoelectronics, Military University of Technology, 00-908 Warsaw, Poland

${ }^{3}$ Faculty of Pharmacy, Wroclaw Medical University, 50-556 Wroclaw, Poland

\begin{abstract}
The attenuated total reflection technique in combination with the terahertz technique is being investigated to find rotatable bonds, which are considered to be one of the rules of thumb given by Veber and others. The results were obtained using a pulsed terahertz spectrometer. Two forms of samples were used to check the methodology: polycrystalline, and saturated solutions in ethyl alcohol. Histidine, as one of the best-studied pharmacological preparations, was used in the experiment to test our research. Four spectral details were found in the terahertz band, including one for which intermolecular vibrations are responsible, and three others for which intramolecular vibrations, such as rotatable bonds, may be responsible. Histidine molecules that are dissolved in alcohol, and thus free of intermolecular bonds, reveal rotations around three bonds. The research methodology presented in the article can support pharmacological research and the pharmaceutical industry.
\end{abstract}

Keywords: pharmacology, histidine, $\mathrm{THz}$ spectroscopy, rules of thumbs

The terahertz technique is mainly applied in biomedicine, and it contributes to the development of scientific disciplines such as pharmacy (1-5). The main goal of pharmaceutical science is the search for effective drugs. All scientific methodologies and technologies are committed to this goal $(6,7)$. It is known that the effectiveness of a drug in the body depends on its amount and the speed of its absorption. The concept that quantitatively characterizes the absorption process is bioavailability $(8,9)$. In order to determine the bioavailability of a compound, a number of practical rules that support drug discoveries, the so-called rules of thumbs, were formulated. The rules of thumbs for selecting "drug-like" compounds cover a wide range of properties, such as the number of rotatable bonds (ROTB), the number of aromatic rings (AROM), the number of hydrogen bond donors and acceptors (HBD and HBA), the polar surface area (PSA) or topological polar surface area (TPSA) [angstroms squared], the acid dissociation coefficient (pKa), lipophilicity ( $\log \mathrm{P}$ and $\log \mathrm{D})$, molecular weight (MW) [moles], and the fraction of sp3 carbons (FSP3) (10). The most famous rule is Lipinski's Rule of Five, also known as Pfizer's Rule of Five, or simply the Rule of Five (RO5). The following criteria of properties were proposed in the RO5: lipophilicity $(\log \mathrm{P})<5$, molecular weight $(\mathrm{MW})<500$, the number of hydrogen bond donors $(\mathrm{HBD})<5$, and the number of hydrogen bond acceptors (HBA) $<10$ (11).

Ghoes et al. provided an analysis of some computable physicochemical properties and the chemical composition of known drug molecules that are available in the Comprehensive Medicinal Chemistry (CMC) database, as well as in seven known drug classes (12). The study showed that the qualifying range of the calculated $\log \mathrm{P}$ for drug-like molecules is -0.4 to 5.6 ; the mean value is 2.3 , and the preferred range (most populated for an interval having $50 \%$ of the drugs) is 1.3 to 4.1 ; for molar refractivity, the qualifying range is 40 to 130 . The mean is 97 , and the preferred range is 70 to 110 ; for molecular weight, the qualifying range is 160 to 480 ; the mean molecular weight is 360 , and the preferred range is 230 to 390 ; for the total number of atoms, the mean value is 48 , and the qualifying range is 20 to 70 . The preferred range for the total number of atoms is 30 to 55 (12). In turn, the Muegge method in the assessment of bioavailability analyses a much larger number of parameters. According to the author's assumptions, a drug with good bioavailability meets the following requirements with regards to its chemical structure: $200 \leq$ molecular weight $(\mathrm{MW}) \leq 600 ;-2 \leq$ lipophilicity $(\log \mathrm{P}) \leq 5$; polar surface area $(\mathrm{PSA}) \leq 150$; number of rings $\leq 7$; number

* Corresponding author: e-mail: edward.plinski@pwr.edu.pl 
of carbons $>4$; number of heteroatoms $>1$; number of rotatable bonds $(\mathrm{ROTB}) \leq 15$; number of hydrogen bond acceptors $(\mathrm{HBA}) \leq 10$; and number of hydrogen bond donors $(\mathrm{HBD}) \leq 5(13)$. In order to achieve the goal more effectively, Congreve limited the RO5 to the rule of three (RO3): number of rotatable bonds $($ ROTB $) \leq 3$; molecular weight $<300$; number of hydrogen bond donors $\leq 3$; number of hydrogen bond acceptors $\leq 3 ; \log \mathrm{P} \leq 3$; and polar surface area $(\mathrm{PSA}) \leq 60$ (14). Additionally, these rules include the following expectations with regards to the beneficial properties of drugs: high activity and selectivity, synthetic availability, no chemically reactive groups, the possibility of oral administration, pharmacokinetic properties, metabolism, routes of removal from the body, and no side effects and toxicity (10).

Even a cursory analysis of the above points, and also research conducted with regards to the above guidelines showed that finding simple rules for the behavior of a drug molecule in a living organism is impossible. All this has prompted scientists to search for new solutions, methods, theories of designing, and technologies for the effective production of drugs. Matthew Segall made an attempt to find a simple rule, based on simple properties that could be used to identify the right ingredients with a much greater chance of creating an effective drug (10). Segall concluded that no single principle could fulfill this role. The closest to the ideal seems to only be the two Veber rules (15): 10 or fewer rotatable bonds (optimally 7), a polar surface area less than or equal to $140 \AA^{2}$ (optimally between $110 \AA^{2}$ and $140 \AA^{2}$ ). The first rule is based on Veber's observations that excessive amounts of rotatable bonds can make it difficult to anchor a drug to the diseased molecule.

When comparing the principles given by Muegge, Congreve, and Veber, the latter ones have the strongest "spectral" character. This is due to the fact that $50 \%$ of Veber's principles can be confirmed experimentally by spectroscopic methods. We mean the detection of rotatable bonds. (We would like to explain that in subject literature, the terms torsion or rotations are given (16). In this paper, we use the term rotations, as torsions are part of rotations). The rotation/torsion frequencies of the molecules are scattered over a fairly wide band of the electromagnetic wave spectrum, but in the case of "heavy" molecules, such as those used in drug design, they are located in the far-infrared (FIR) part of the spectrum, which is known today as the terahertz (THz) band (17-20). The aim of this study is to demonstrate the relationship between pharmacological rules of thumbs and the terahertz technique.

One of the amino acids - histidine - was selected for the experiment (21). The histidine molecule is composed of an imadosyl ring, a carboxyl group, and an amino group (see Figure1). It can be found in two forms: the L-histidine (the L-enantiomer of the amino group), or the L-histidine zwitterion (the zwitterionic form of the L-histidine has an anionic carboxy group and a protonated $\alpha$-amino group). "L" means leftoriented in the Fisher projection (22). The first two components of the molecule are linked by the three single bonds $\mathrm{C} 1-\mathrm{C} 2, \mathrm{C} 2-\mathrm{C} 3$, and $\mathrm{C} 3-\mathrm{C} 4$. According to the definition of Veber: "Rotatable bonds were defined as any single bond, not in a ring, and bound to a nonterminal heavy (i.e., non-hydrogen) atom" (15). Therefore (in the case of histidine), a rotation around the three mentioned bonds, as shown in the figure, can be expected. We also expect that for sufficiently "heavy" molecules like histidine, the frequencies of the measured vibrations will be in the far infrared or, in other words, in the terahertz band. If spectroscopy will be conducted on a powdered sample, all possible vibrations, both intermolecular and intramolecular (including rocking/bending/wagging vibrations and rotations/torsions of molecules) will be seen - see Figure 2.

The figure shows a hypothetical lattice in which histidine molecules are trapped in nodes. Even from such a simplified drawing, it can be seen that the released molecules from the crystallographic lattice should only exhibit torsional/rotational or rocking/bending/waging movements. This observation leads directly to the methodology for designing an appropriate experimental setup. In our opinion, samples should be tested in such conditions in which they are released from the crystallographic lattice bonds.
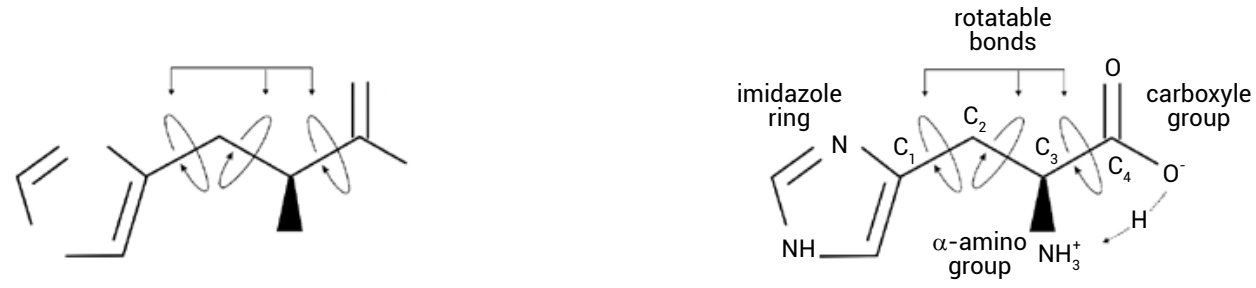

Figure 1. Molecule of histidine: left - L-histidine, right - L-histidine zwitterion. Possible. 
The histidine crystal lattice belongs to the space group $\mathrm{P} 2{ }_{1}$ (monoclinic space group), or $\mathrm{P} 22_{1} 2_{1}$ (orthorhombic) (23-25)). Possible intermolecular vibrations and torsional movements are indicated. In the case of the polycrystalline form of the sample, both translational and rotational vibrations can be observed in the terahertz spectrum. On the other hand, an attempt to test a dissolved sample, i.e. one with no lattice bonds, presents other difficulties. Solvents, starting with water, are characterized by strong absorption properties for terahertz waves. Therefore, the appropriate research system is a terahertz spectrometer equipped with a Dove prism, where the phenomenon of the so-called evanescent wave penetrating the sample to a small depth of several to several dozen micrometers is used. This is called the methodology of ATR (Attenuated Total Reflection) spectroscopy (26-30). A different system, which uses the classical transmission technique, but in the system of rotatable cells with samples, is also possible (31).

\section{EXPERIMENTAL}

A system that allows for $\mathrm{THz}$ liquid spectroscopy, also with the possibility of testing powdered samples, was prepared for the tests. This can be seen as a convenient solution. A classic Time Domain spectroscopy (THz-TDS) experimental set, driven by a pulsed femtosecond laser ( $83 \mathrm{fs}$ ) with a repetition frequency of $80 \mathrm{MHz}$ and a wavelength of $780 \mathrm{~nm}$, was used. Terahertz radiation was created by a photoconductive antenna made of LT-GaAs (low temperature of gallium arsenide). The same type of photo antenna was used as the detector. The terahertz beam was formed and focused using parabolic

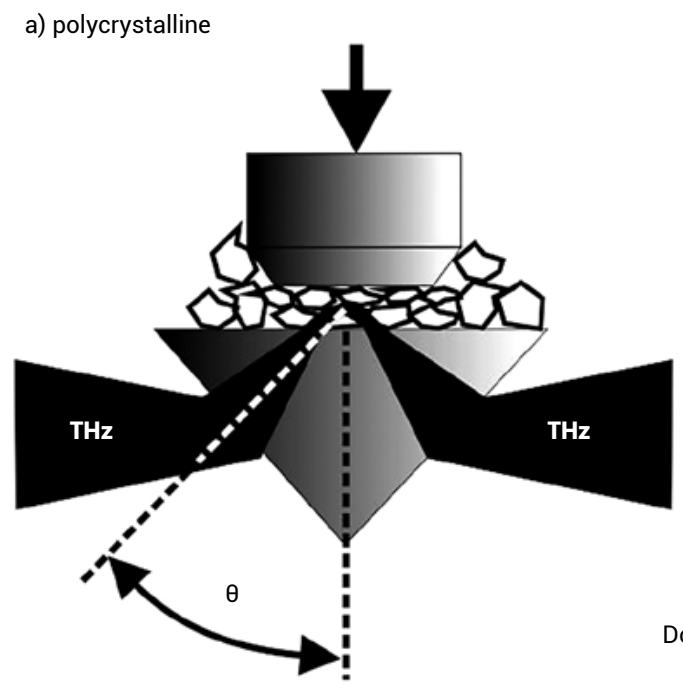

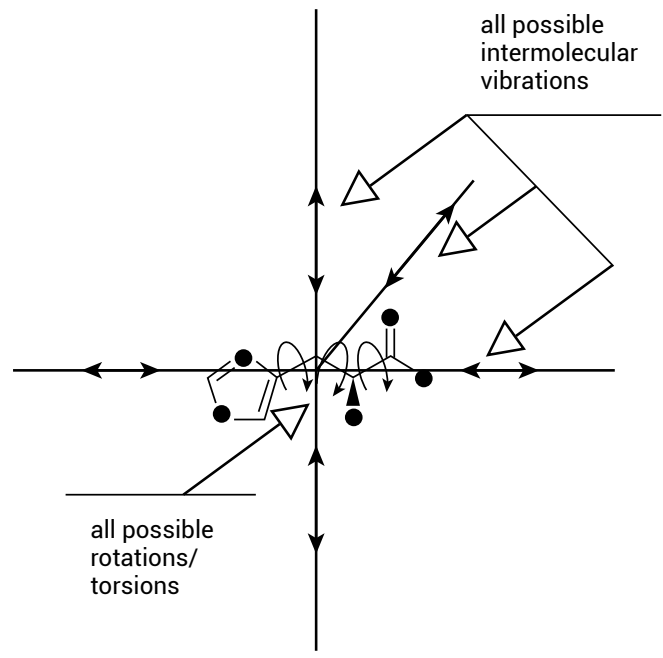

Figure 2. Schematic drawing of one of the nodes of the crystal lattice in which the histidine molecule is trapped. Possible molecular vibrations, both intermolecular and intramolecular, are marked.

off-axis mirrors $(5,18)$. A Dove prism made of high resistivity silicone (appr. $10 \mathrm{k} \Omega \mathrm{cm}$ ) was placed in the path of the $\mathrm{THz}$ measuring beam. The Dove prism is an optical element prepared in such a way that the beam of terahertz radiation passing through it does not change its direction, i.e. the transmitter-receiver path remains unchanged (see Figure 3).

The histidine samples were purchased from Sigma-Aldrich (molecular weight of $155.15 \mathrm{~g} /$ $\mathrm{mpl}$ ). The samples were tested in two forms: polycrystalline, and in the form of saturated solutions in anhydrous ethyl alcohol 99.8\% ( $\mathrm{Ph} \mathrm{65).}$ The polycrystalline samples were not prepared in any particular way. The material in the SigmaAldrich packaging was used. All the samples

b) sample solved

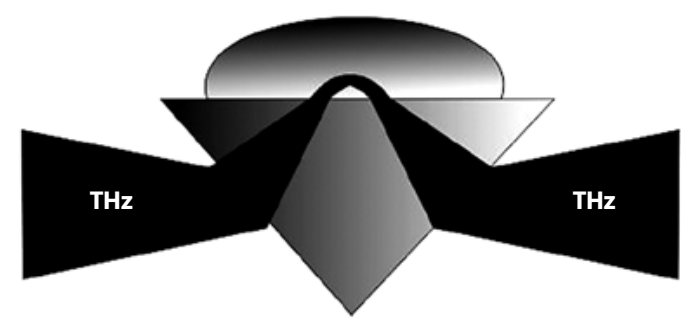

Dove prisms

Figure 3. Attenuated Total Reflection (ATR) spectroscopy. a) investigations of polycrystalline forms. The method of pressing against the prism's surface is shown. The incident angle is also indicated. b) investigations of solved samples. 
were placed on the surface of the prism. The entire path of the terahertz beam, i.e. the entire measuring portion from the transmitter to the receiver, was enclosed in a plastic vessel that was blown through with dry air (less than $20 \mathrm{ppb}$ moisture).

The experiment used the technique of attenuated total reflection (ATR) (24-28). In terms of measuring the spectrum of molecules released from intermolecular bonds, the ATR method is a convenient solution. The ATR technique uses the phenomenon of total internal reflection, in which the evanescent wave (EV) develops along the boundary surface of the prism. The phenomenon of the evanescent wave occurs when the incident wave passes through the medium (here Si crystal) with a higher refractive index (here ca. 3.41) to the tested sample with a lower refractive index (here ca. 1.3).

The penetration depth of the EV into a sample is relatively small. It depends on the angle of incidence of the radiation beam and its polarization. The spectral measurements were carried out on the apparatus of TereView Ltd. Hence, the parameters mentioned above were established by the manufacturer. The disclosed data are the refractive index of the material from which the Dove prism (Si crystal) was made, 3.41, and the angle of incidence $\theta=35^{\circ}$. For these data, we can calculate the penetration depth $d_{p}$ of the evanescent wave (32):

$$
d_{p} \approx \frac{\lambda}{2 \pi \sqrt{n_{1}^{2} \sin ^{2} \theta-n_{2}^{2}}}
$$

where: $\theta$ - incident angle for the THz wave, $n_{1}$ - refractive index of the ATR (Dove) crystal,

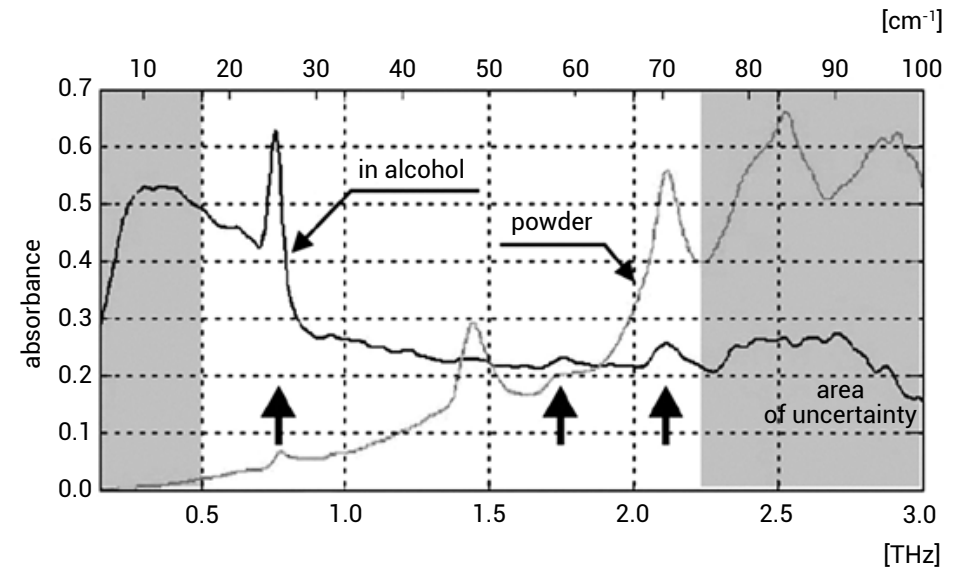

Figure 4. Experimental results of the terahertz spectroscopy of the histidine samples in both powder form and when dissolved in alcohol. Arrows mark the spectral details for which rotations/torsions around the rotatable bonds of the molecule may be responsible. (Areas of uncertainty are indicated in the picture) $n_{2}$ - refractive index of the investigated media,

$\lambda$ - the wavelength.

Assuming the refractive index of the tested samples approx. $n_{2}=1.3$ and $n_{1}=3.4$ for a high resistivity silicon crystal, we obtain the penetration depth from approx. $240 \mu \mathrm{m}$ to $24 \mu \mathrm{m}$ depending on the frequency of the incident wave, from $0.3 \mathrm{THz}$ to $3 \mathrm{THz}$ (from $1000 \mathrm{~m}$ to $100 \mathrm{~m}$ ), respectively. The exact value depends on the wavelength of the probe beam, the angle of incidence, and the refractive indexes. The polarization of the incident beam also affects the penetration depth. The above calculations were performed for the incident beam perpendicular to the surface of the Dove crystal. Tu należy wiedzieć, że dla polaryzacji równoległej wyniki mogą być dwa razy większe (32). Here it should be known that for parallel polarization the results can be twice as large (32). Even a rough estimation of the depth of penetration is information for the researcher - it may be insufficient for the wave to penetrate the molecules of the tested medium. Hence, in the case of the powdered samples, the sample was additionally pressed against the prism's surface in order to ensure good contact and also to remove trapped air that could reduce the intensity of the signal (in the case of the TeraView apparatus it was $20 \mathrm{~kg}$ on $7 \mathrm{~mm}$ in diameter of the investigated sample). The experiment was prepared in the same measurement ATR system for the samples in polycrystalline form and the samples dissolved in ethyl alcohol (as can be seen in Figure 3, left and right, respectively). A significant advantage of the ATR method is the ability to measure media that strongly absorb the terahertz wave. Another advantage also appears when measuring polycrystalline samples. Scientists who often deal with the synthesis of molecules (drugs) receive only small amounts of the expected material. The ATR method enables this disadvantage to be overcome. This is due to the fact that $1 \mathrm{mg}$ of the synthesized molecules is sufficient for successful measurements.

\section{RESULTS}

\section{Histidine}

The results of the THz-TDSATR spectroscopy are shown in the graph in Figure 4. The graph is prepared for two systems of units: frequency in terahertz $(\mathrm{THz})$, and in reciprocal centimeters $\left(\mathrm{cm}^{-1}\right)$. In 
Table 1. Results of the histidine terahertz spectrum measurements.

\begin{tabular}{|c|c|c|c|c|c|}
\hline \multicolumn{4}{|c|}{ Spectral details } & Units & References \\
\hline $0.70(0.68)$ & 1.41 & 1.77 & 2.19 & \multirow{5}{*}[\mathrm{THz}]{} & (this work) \\
\hline 0.76 & & & 2.07 & $(16)$ \\
\hline 0.78 & 1.42 & 1.74 & 2.11 & $(33)$ \\
\hline 0.77 & & 1.72 & 2.08 & & $(34,35)$ \\
\hline $23.35(22.68)$ & 47.03 & 59.04 & 73.05 & \multirow{5}{*}[\mathrm{cm}^{-1}]{} & $($ this work) \\
\hline 25.35 & & & 69.00 & $(16)$ \\
\hline 26.02 & 47.37 & 58.04 & 70.38 \\
\cline { 3 - 4 } \cline { 3 - 4 } & & 57.40 & 69.46 & & $(33)$ \\
\hline 25.56 & & & & & \\
\hline
\end{tabular}

the polycrystalline sample, all possible vibrations of the molecule can be observed.

As can be seen, the dissolved sample shows a strong absorption peak at $0.74 \mathrm{THz}\left(24.68 \mathrm{~cm}^{-1}\right)$, and two weaker peaks at $1.73 \mathrm{THz}\left(57.71 \mathrm{~cm}^{-1}\right)$ and $2.12 \mathrm{THz}\left(70.72 \mathrm{~cm}^{-1}\right)$ - indicated by arrows. The results are presented in Table 1.

As can be seen in the table, the frequencies of the spectral details are practically the same, whether the sample is polycrystalline or dissolved in alcohol. There is one exception: we observed a slight drift in the frequency of the strongest peak around $0.70 \mathrm{THz}\left(23.35 \mathrm{~cm}^{-1}\right)$ towards the lower frequencies of $0.68 \mathrm{THz}\left(22.68 \mathrm{~cm}^{-1}\right)$ for the sample dissolved in alcohol - see the values in parentheses in Table 1 . The peak around $1.41 \mathrm{THz}\left(47.03 \mathrm{~cm}^{-1}\right)$ practically disappears in the case of the dissolved sample, i.e. without lattice bonds.

a)

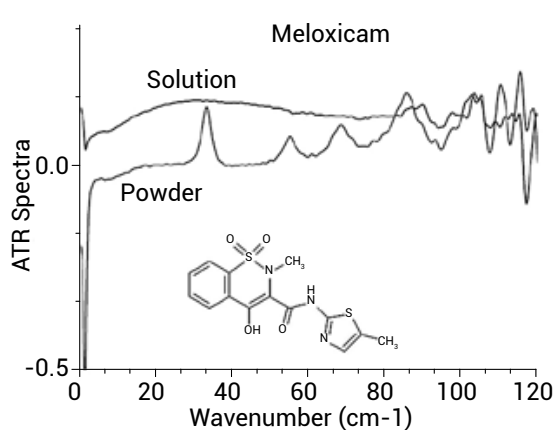

c)

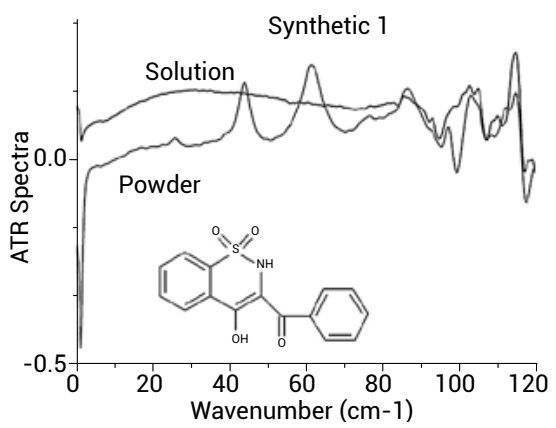

The spectrum of the dissolved substance returns to its former shape when the ethanol is evaporated. The spectrum obtained corresponds to the original spectrum, i.e. for the sample in a crystalline form.

\section{Other selected samples.}

To illustrate the scale of the difficulty of the idea of detecting rotating bonds presented here (so important for the pharmacological rules of thumbs), additional measurements were performed for a few selected molecules (see Figure 5) (36). The selected molecules can be described as "pharmacologically oriented" including known molecules like meloxicam and piroxixam and two synthesized once.

The measurement methodology is described above. According to (37), for preparation a), 3 rotatable bonds are predicted, for $b$ ) - 3 rotatable bonds, for c) - 2 rotatable bonds, for d) - 3 rotatable bonds.

b)

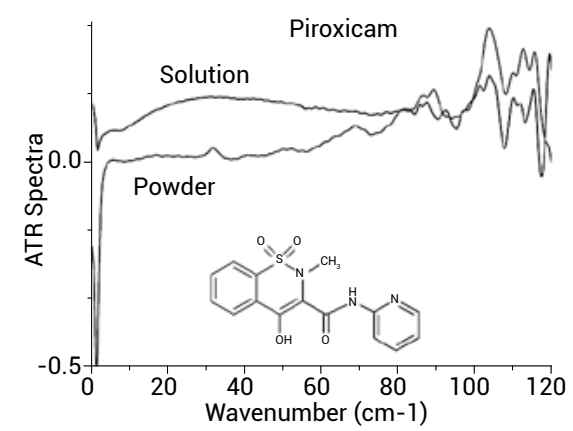

d)

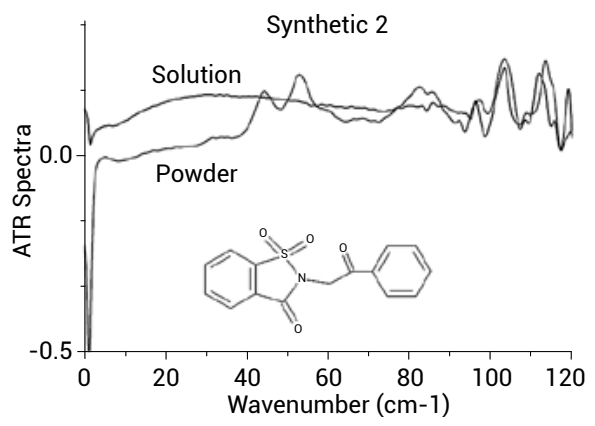

Figure 5. Selected molecules on which ATR tests were carried out: a) meloxicam, b) piroxicam, and two synthesized samples, c) synthetic $1, \mathrm{~d})$ synthetic $2,(36)$. 
As it is seen, each test sample in powder form (in a crystalline state) shows clear spectral details. However, none of the samples show any distinct spectral details in the dissolved state. This experiment shows the scale of difficulties in experimental detecting (confirming) rotatable bonds using terahertz ATR spectroscopy.

\section{DISCUSSION AND CONCLUSION}

As expected, the diagrams in Figure 4 show all possible spectral details, which are caused by both the intermolecular vibrations and internal movements of the histidine molecule in the terahertz range of the spectrum. The true picture of the molecule is revealed when it is free from intermolecular vibrations. Then, only the internal movements of the molecule can be observed - the peak at $1.41 \mathrm{THz}\left(47.03 \mathrm{~cm}^{-1}\right)$ disappears. (This spectral detail is probably due to intermolecular vibrations - see Figure 2 and 3, and Table 1). In the molecule freed from crystallographic lattice bonds, both rocking/bending/wagging vibrations and rotations/torsions can be observed. The determination of the type of vibrations can be derived from the analysis of the structure of the histidine molecule (see Figure 1). According to Veber: "Rotatable bonds were defined as any single bond not in a ring ..." (15). This means that rotatable bonds can be expected around the three single bonds $\mathrm{C} 1-\mathrm{C} 2, \mathrm{C} 2$ $\mathrm{C} 3$, and $\mathrm{C} 3-\mathrm{C} 4$. Another suggestion may be derived from the research of True (33). The author gives all four spectral details for histidine. However, they are not assigned to any kind of molecular vibrations, but what should be emphasized here is that the samples were only tested in polycrystalline form. Another work that sheds light on the types of vibrations in the histidine molecule is the work of Matei (16). The paper indicates the possible vibrations of amino acids, such as rocking/wagging/bending modes and torsions. Matei calls the peak around $0.75 \mathrm{THz}\left(25.02 \mathrm{~cm}^{-1}\right)$ "torsions of the entire side chain about the C-ring bond." On the other hand, Matei assigns the peak at $7.07 \mathrm{THz}\left(69 \mathrm{~cm}^{-1}\right)$ to the hydrogen bond mode. It should be again emphasized here that the studies were only carried out on powdered samples. The paper of Rungsawang experimentally confirms the existence of the three spectral details $0.77,1.72$, and $2.08 \mathrm{THz}(25.56,57.40$, and $69.46 \mathrm{~cm}^{-1}$ ) in the terahertz spectrum. These frequencies were also observed in our experiment. The samples were also tested only in polycrystalline form (34-35). In the paper of Yi, it was noted that some amino acids, like L lysine and L-arginine, exhibit common absorption spectra details at 0.90 , 1.44, 2.07, and 2.56 THz (30.02, 48.03, 69.05, and $85.39 \mathrm{~cm}^{-1}$ ) due to the similarity of their structures. However, these vibrations are not associated with any bonds. The results were also obtained only for the powdered sample (19). Taking into account the results of our experiment, it is highly probable that in the work of $\mathrm{Yi}$, intermolecular vibrations are responsible for the peak at $1.44 \mathrm{THz}\left(48,03 \mathrm{~cm}^{-}\right.$ $\left.{ }^{1}\right)$ in lysine and arginine. Similarly, in the work of Yamamoto, a polyglycine absorption peak at $1.36 \mathrm{THz}\left(45.5 \mathrm{~cm}^{-1}\right)$ was assigned to interchain vibrations (not intrachain!). Their result was also only obtained experimentally on a powdered sample (17). On the other hand, the Guide for Pharmacology, SwissADME, and PubChem clearly indicate the existence of three rotatable bonds in the histidine molecule (37). However, the guides do not assign them to any specific bonds. It should be emphasized that to our knowledge none of the available studies present the results of histidine tests performed in any solvent environment. Moreover, despite the research conducted on powdered samples - polycrystalline - only one study gives a spectral detail of around $1.42 \mathrm{THz}\left(47.37 \mathrm{~cm}^{-1}\right)$ (33).

It is this absorption peak that disappears after dissolving the histidine in the solvent, which may indicate that this peak is caused by the intermolecular vibrations between the nodes of the crystallographic lattice (to be compared with the paper of $\mathrm{Yi}-1.44 \mathrm{THz}\left(48.03 \mathrm{~cm}^{-1}\right)$ in lysine and arginine). In addition, we noticed that the frequencies of the absorption peaks may vary depending on whether the sample was powdered or dissolved in ethanol. In the conditions of crystallographic lattice bonds for one of the peaks, we obtained the frequency of $0.70 \mathrm{THz}\left(23.35 \mathrm{~cm}^{-1}\right)$, and in the solvent medium, we obtained the frequency of $0.68 \mathrm{THz}\left(22.68 \mathrm{~cm}^{-1}\right)$.

Here it should be mentioned about a suitable correction of the ATR spectrum. In the range of the obtained spectrum, the sample parameters, such as the dielectric constant, are variable, and this affects the refractive index of the tested material. This effect changes the shape of the studied spectrum. There are various more or less complex algorithms for the correction of the results obtained by ATR spectroscopy $(38,39)$. In our experiment, the ATR correction algorithms were integrated into the TeraView software we used. Besides, our goal was not to quantify spectral data in terms of the levels of the spectral curves. The main aim of the study was to search for spectral details responsible for the intra- and intermolecular vibrations of the examined medium. More specifically, the aim was 
to determine the position of the absorption peaks within the available terahertz spectrum as shown in Table 1. Although it is very likely, that observed a slight drift in the frequency: $0.70 \mathrm{THz}\left(23.35 \mathrm{~cm}^{-}\right.$ $\left.{ }^{1}\right)$ towards $0.68 \mathrm{THz}\left(22.68 \mathrm{~cm}^{-1}\right)$ - see Table 1 - is so-called "redshift", a typical phenomenon as a consequence of the considerations above. On the other hand, the difference was only about $0.02 \mathrm{THz}$ $\left(0.67 \mathrm{~cm}^{-1}\right)$, and the resolution of the TeraView equipment had a value twice as high $(0.04 \mathrm{THz})$. As you can see, ATR methodology practically does not change much the positions of the spectral peaks in the spectrum.

For the same reasons, we ignored the influence of the ethyl alcohol in which the samples were dissolved. The spectrum of ethyl alcohol is smooth and shows no spectral details within our research range up to $3.6 \mathrm{THz}$ ((40) - Figure 2a). The absorption coefficient varies in this range from $35 \mathrm{~cm}^{-1}$ to $100 \mathrm{~cm}^{-1}$, but due to its even increment, it does not affect the spectral details of the tested substance. It is known that rotatable bonds are taken into account as one of the rules of thumbs given by e.g. Veber et al. It is also known that the THz technique is a sensitive and selective technique that enables the detection and identification of the details of the molecular structure (16-20). Within the terahertz band, vibrations of molecules, such as rocking, bending, wagging, rotations/torsion, and intermolecular vibrations, can be observed. The analysis of the molecular structure of the histidine molecule (see Figure 1), data in chemical guides (37), and also Veber's considerations, lead to the conclusion that spectroscopic studies of the histidine molecule within the terahertz band in a solvent environment can result in spectral details for which only rotations/torsions are responsible - the so-called rotatable bonds. This is the link we are looking for between the rules of thumbs and the $\mathrm{THz}$ technique. The results of other authors, in which some vibrations are assigned to hydrogen bond torsions, should also be taken into account (16). Moreover, our observations show that the frequencies of some spectral peaks may differ depending on whether the sample was tested in a powdered form or whether it was dissolved in alcohol, i.e. without lattice bonds. The methodology of computational chemistry was not the purpose of this work. Although taking into account what has been said in the discussion of the results, computational chemistry may provide a final solution to the problems mentioned above. The above considerations and results also lead to a conclusion that may direct the experimental technique: in our opinion, experimental studies of pharmacological samples for the purpose of confirming the recommendations indicated by the so-called rules of thumbs should be carried out in solvent conditions (alcohol, chloroform, etc.). Finally, the methodology for detecting rotational bonds described above comes down to the "click-clack" technique, that is, comparing the results for the solid form with the results for the dissolved form of the test material. Due to the strong absorption of solvents for terahertz waves, the THzTDS-ATR research methodology is recommended. The effectiveness of this method has been shown and is the main result of this paper: the demonstration of the participation of the THz-TDS-ATR terahertz spectroscopy technique in the detection of rotatable bonds, the amount of which is important in the design of drug-like candidate compounds. However, the methodology presented in this paper does not always produce the expected results. Spectral bands resulting from intramolecular vibrations often overlap, masking each other. This effect occurs for molecules more complex than the histidine considered here, as shown in Figure 5. It is certainly necessary to develop more sensitive apparatus and new research methods. (Here we should mention transmission technology applied to liquids (31). This experiment uses a system, which uses the classical transmission technique, but in the system of rotatable cells with samples). In our opinion, this result does not undermine the methodology of searching for rotatable bonds described in detail in the paper. As it is known, the number of rotatable bonds may be crucial for the acceptance of a drug candidate, which is clearly distinguished in the rules of thumbs given by Muegge, Congreve, and particularly Veber, where the latter gives as much as $50 \%$ of the importance of the formulated rules to rotatable bonds. Considering the above, the research methodology presented in the article may bring tangible benefits for pharmacological research, and thus support the pharmaceutical industry.

\section{Acknowledgments}

The authors would like to acknowledge Professor Wieslaw Malinka and Dr. Berenika Szcześniak-Sięga from Wroclaw Medical Academy for fruitful discussions about the pharmacology ideas. The authors are grateful to Przemyslaw Jarząb and Kacper Nowak for their technical support.

\section{Conflicts of interest}

The authors declare that there are no conflicts of interest. 


\section{REFERENCES}

1. Taday P.F.: Philos. Trans. A Math. Phys. Eng. Sci. 362, 351 (2004).

2. Markl D., Ruggiero M.T., Zeitler J.A.: Eur. Pharm. Rev. 21, 45 (2016).

3. Sterczewski L.A., Nowak K., Szlachetko B., Grzelczak M.P., Szczesniak-Siega B., et al.: Sci. Rep. 7, 14583 (2017).

4. Gong A., Qiu Y., Chen X., Zhao Z., Xia L., Shao Y.: Appl. Spectrosc. Rev. 55, 418 (2019).

5. Pickwell E, Wallace V.P.: J. Phys. D Appl. Phys. 39, R301 (2006).

6. Shen, Y.C.: Int. J. Pharm.: 417, 48 (2011).

7. Parrott E.P., Zeitler J.A.: Appl. Spectsc. 69, 1 (2015).

8. Currie G.M.: J. Nucl. Med. Technol. 46, 221 (2018).

9. Egan W.J., Merz K.M., Baldwin J.J.: J. Med. Chem. 43, 3867 (2000).

10. Segall M.: Drug Disc. 15, 19 (2016).

11. Lipinski C.A., Lombardo F., Dominy B.W., Feeney P.J.: Adv. Drug Deliv. Rev. 46, 3 (2001).

12. Ghose A.K., Viswanadhan V.N., Wendoloski J.J.: J. Comb. Chem. 1, 55 (1999).

13. Muegge I.: Chemistry 8, 1976 (2002).

14. Congreve M., Carr R., Murray C., Jhoti H.: Drug Discov. Today 8, 876 (2003).

15. Veber D.F., Johnson S.R., Cheng H.Y., Smith B.R., Ward K.W., Kopple K.D.: J. Med. Chem. 45, 2615 (2002).

16. Matei A., Drichko N., Gompf B., Dressel M.: Chem. Phys. 316, 61 (2005).

17. Yamamoto K., Tominaga K., Sasakawa H., Tamura A., Murakami H., et al.: Biophys. J. 89, L22 (2005).

18. Nowak K., Plinski E.F., Karolewicz B., Jarzab P.P., Plinska S., et al.: Acta Pol. Pharm. 72, 851 (2015).

19. Yang X., Zhao X., Yang K., Liu Y., Liu Y., et al.: Trends Biotechnol. 34, 810 (2016).

20. Yi W., Yu J., Xu Y., Wang F., Yu Q., et al.: Instrum. Sci. Tech. 45, 423 (2017).

21. Kulis-Horn R.K., Persicke M., Kalinowski J.: Microb. Biotechnol. 7, 5 (2014).
22. McMurry J.E.: Organic Chemistry, Brooks/Cole, 2007.

23. Suresh C.G., Vijayan M.: Int. J. Pept. Protein Res. 22, 129 (1983).

24. Madden J.J., McGandy E.L., Seeman N.C., Harding M.M., Hoy A.: Acta Cryst. B28, 2382 (1972).

25. Madden J.J., McGandy E.L., Seeman N.C., Harding M.M., Hoy A.: Acta Cryst. B28, 2377 (1972).

26. Hirori H., Yamashita K., Nagai M., Tanaka K.: Jap. J. Appl. Phys. 43, L1287 (2004).

27. Nagai M., Yada H., Arikawa T., Tanaka K.: Int. J. Infrared Milli. 27, 505 (2006).

28. Soltani A., Jahn D., Duschek L., Castro-Camus E., Koch M., Withayachumnankul W.: IEEE Trans. Terahertz Sci. Technol. 6, 32 (2016).

29. Minnes R., Nissinmann M., Maizels Y., Gerlitz G., Katzir A., Raichlin Y.: Sci. Rep. 7, 7 pages (2017).

30. Huang Y., Singh R., Xie L., Ying Y.: Appl. Sci. 10,18 pages (2020).

31. Balakrishnan J.M., Fischer B.M., Abbott D.: IEEE Photonics J. 1, 88 (2009).

32. Newnham D.A., Taday P.F.: Appl. Spectrosc. 62, 394 (2008).

33. True A.B., Schroeck K., French T.A., Schmuttenmaer Ch.A.: J. Infrared Millim. Terahertz Waves 32, 691 (2011).

34. Rungsawang R., Ueno Y., Tomita I., Ajito K.: J. Phys. Chem. B. 110, 21259 (2006).

35. Ueno Y., Rungsawang R., Tomita I., Ajito K.: Anal. Chem.78, 5424 (2006).

36. Szczęśniak-Sięga B.M., Mogilski S., Wiglusz R.J., Janczak J., Maniewska J., et al.: Bioorg. Med. Chem. 27, 1619 (2019).

37. https://www.guidetopharmacology.org; http:// www.swissadme.ch/index.php; https://pubchem. ncbi.nlm.nih.gov/compound/Histidine (accessed on 10.04.2021).

38. Huang J.B., Urban M.W.: Appl. Spectrosc.: 46, 1666 (1992).

39. Bertie J.E., Eysel H.H.: Appl. Spectrosc.: 39, 392 (1985).

40. Sarkar S., Saha D., Banerjee S., Mukherjee A., Mandal P.: Chem. Phys. Lett.: 678, 65 (2017). 